\title{
On the linearization stability of the Chern-scalar curvature
}

\author{
Daniele Angella ${ }^{1} \cdot$ Francesco Pediconi $^{1}$
}

Received: 28 June 2021 / Accepted: 10 December 2021 / Published online: 24 January 2022

(c) The Author(s) 2022

\begin{abstract}
In this note, we study the local properties of the Chern-scalar curvature function by looking at its linearization. In particular, we study its linearization stability and the structure of the space of Hermitian metrics with prescribed Chern-scalar curvature.
\end{abstract}

Keywords Hermitian manifold · Chern connection · Chern scalar curvature · Linearization stability

Mathematics Subject Classification 53C21 · 53C55

\section{Introduction}

In Riemannian geometry, the space of Riemannian metrics $\mathcal{M}$ attached to a differentiable manifold $M$ plays a crucial role as a differentiable invariant. From this point of view, the function scal, assigning to any Riemannian metric $g$ its scalar curvature $\operatorname{scal}(g)$, is well understood. We refer e.g. to the seminal works [6,21-24]. For compact surfaces $S$, the image of this function depends on the Euler characteristic. Indeed, the necessary and sufficient conditions for a smooth function to be the Gaussian curvature of some metric are either to be positive somewhere when $\chi(S)>0$, or to change sign or to be identically zero when $\chi(S)=0$, or to be negative somewhere when $\chi(S)<0$, see [21, Thm 6.3, Thm 11.8], [23, Thm 5.6]. There is a similar trichotomy in higher dimension, where one makes advantage of the sign of the first eigenvalue of the conformal Laplacian operator, see [23, Thm 6.4]. Two main ingredients in the work by Kazdan and Warner are the study of the local surjectivity of the map $g \mapsto \operatorname{scal}(g)$ by means of the Inverse Function Theorem [24, Lemma 2, p 228], and an Approximation Lemma for studying the $L^{p}$-closure of the orbits of a function under

The authors are supported by project PRIN2017 "Real and Complex Manifolds: Topology, Geometry and holomorphic dynamics" (code 2017JZ2SW5), and by GNSAGA of INdAM.

Francesco Pediconi

francesco.pediconi@unifi.it

Daniele Angella

daniele.angella@unifi.it,daniele.angella@gmail.com

1 Dipartimento di Matematica e Informatica "Ulisse Dini”, Università degli Studi di Firenze, viale Morgagni 67/a, 50134 Firenze, Italy 
the action of the diffeomorphism group of $M$ [24, p. 228]. This note is born as an attempt to understand similar questions concerning the geometry of Hermitian metrics on a compact complex manifold.

In Hermitian geometry, the Levi-Civita connection is replaced by Hermitian connections with possibly non-zero torsion. Among Hermitian connections, there are some canonical choices (in the sense of [16]), in particular, the Chern connection is uniquely characterized by having torsion of type $(2,0)$, equivalenty, by the $(0,1)$-component coinciding with the Cauchy-Riemann operator of the holomorphic tangent bundle, see e.g. [16, p. 273]. The Chern-scalar curvature $\mathrm{scal}^{\mathrm{Ch}}$, obtained by tracing the curvature of such connection (see Section 2 for the definition), has been investigated by several authors. Hermitian metrics on compact complex manifolds with constant Chern-scalar curvature have been investigated and constructed in $[2,4,26]$ and others. A first result on prescribing the Chern-scalar curvature appear in [20, Thm 1.1], and the problem has been recently addressed in [13] by the conformal methods of Kazdan and Warner.

In this note, we exploit the techniques by $[6,10,12,24]$ to study the relationship between infinitesimal and actual deformations of the Chern-scalar curvature function with respect to a varying metric. Concerning the analogue results for the Riemannian scalar curvature, we refer in particular to [12, Thm A, Thm A', Thm 7.9].

Let $(M, J)$ be a compact, connected, complex manifold of dimension $\operatorname{dim}_{\mathbb{R}} M=2 \mathrm{~m}$, and $\mathcal{M}_{\mathrm{H}}$ the space of smooth Hermitian metrics on it. We study the local properties of the function scal ${ }^{\mathrm{Ch}}: \mathcal{M}_{\mathrm{H}} \rightarrow \mathcal{C}^{\infty}(M, \mathbb{R})$ by looking at its linearization (see Proposition 3.6 and Proposition 3.10). Let us recall that $\mathrm{scal}^{\mathrm{Ch}}$ is said to be linearization stable at a metric $g_{\mathrm{o}} \in$ $\mathcal{M}_{\mathrm{H}}$ if, for any direction $h \in \operatorname{ker}\left(\mathrm{scal}^{\mathrm{Ch}}\right)_{g_{0}}^{\prime}$, there exists a smooth path $g:(-\epsilon, \epsilon) \rightarrow \mathcal{M}_{\mathrm{H}}$ such that $g(0)=g_{\mathrm{o}}, \dot{g}(0)=h$ and $\operatorname{scal}^{\mathrm{Ch}}(g(t))=\mathrm{scal}^{\mathrm{Ch}}\left(g_{\mathrm{o}}\right)$ for any $-\epsilon<t<\epsilon$. On the other hand, if scal ${ }^{\mathrm{Ch}}$ is not linearization stable at $g_{\mathrm{o}}$, it is said to be linearization unstable at $g_{\mathrm{o}}$. Our first result reads as follows:

Theorem A Let $g \in \mathcal{M}_{\mathrm{H}}$, set $\lambda:=\operatorname{scal}^{\mathrm{Ch}}(g) \in \mathcal{C}^{\infty}(M, \mathbb{R})$ and assume that either $g$ is not first-Chern-Einstein, or $g$ is first-Chern-Einstein with $\frac{\lambda}{m}<\mathrm{d}^{*} \vartheta$. Then, the function $\mathrm{scal}^{\mathrm{Ch}}: \mathcal{M}_{\mathrm{H}} \rightarrow \mathcal{C}^{\infty}(M, \mathbb{R})$ is linearization stable at $\mathrm{g}$ and maps any neighborhood of $\mathrm{g}$ onto a neighborhood of $\lambda$.

In the statement of the theorem above, $\vartheta$ denotes the torsion 1-form of $g$ (see Eq. (2.4)). Moreover, the first-Chern-Einstein condition is a generalization of the Kähler-Einstein equation in the Hermitian, possibly non-Kählerian, setting (see Sect. 2). In particular, since $\vartheta=0$ and scal $^{\mathrm{Ch}}=$ scal at any Kähler metric, we remark that this theorem applies to KählerEinstein metrics with negative scalar curvature.

Our second result concerns the structure of the space $\mathcal{M}_{\mathrm{H}}(\lambda):=\left\{g \in \mathcal{M}_{\mathrm{H}}: \operatorname{scal}^{\mathrm{Ch}}(g)=\right.$ $\lambda\}$ of Hermitian metrics with prescribed Chern-scalar curvature. More precisely, we prove

Theorem B Let $\lambda \in \mathcal{C}^{\infty}(M, \mathbb{R})$ and assume that $\mathcal{M}_{\mathrm{H}}(\lambda)$ is not empty. If either $(M, J)$ is non-Kählerian and $c_{1}^{\mathrm{BC}}(M, J) \neq 0$, or $(M, J)$ is Kählerian and $c_{1}(M, J)$ has no sign, then $\mathcal{M}_{\mathrm{H}}(\lambda)$ is a closed smooth ILH-submanifold of $\mathcal{M}_{\mathrm{H}}$ with tangent space $T_{g} \mathcal{M}_{\mathrm{H}}(\lambda)=$ $\operatorname{ker}\left(\mathrm{scal}^{\mathrm{Ch}}\right)_{g}^{\prime}$ at $g \in \mathcal{M}_{\mathrm{H}}$.

Here, we say that $(M, J)$ is Kählerian if it admits Kähler metrics, non-Kählerian otherwise. We also denoted by $c_{1}^{\mathrm{BC}}(M, J)$ and $c_{1}(M, J)$ the first Chern class of $(M, J)$ in the Bott-Chern and de Rham cohomology, respectively. Moreover, for the notion of infinite-dimensional ILHmanifold, we refer to [27, Ch II]. Notice that both the hypotheses stated in Theorem B assure that the manifold $(M, J)$ does not admit any first-Chern-Einstein metric, which is a key 
point in the proof. Let us stress also that, while in the Riemannian case the prescribed scalar curvature problem is well understood (see [9, Thm 4.35]), the question whether $\mathcal{M}_{H}(\lambda)$ is non-empty is far from being answered. Therefore, we collect in Remark 4.3 the state of the art, up to our knowledge.

Finally, in our last result, we exhibit concrete examples of Hermitian metrics at which $\mathrm{scal}^{\mathrm{Ch}}$ is linearization unstable. Notice that, by Theorem A, any such metric $g$ is necessarily first-Chern-Einstein with $\frac{1}{m} \mathrm{scal}^{\mathrm{Ch}}(g)-\mathrm{d}^{*} \vartheta \geq 0$ at some point. More precisely, we prove

Theorem C Let $g_{\mathrm{o}}$ be a Kähler-Einstein metric with positive scalar curvature. If $\left(M, g_{\mathrm{o}}\right)$ admits global non-trivial Killing vector fields, then $\mathrm{scal}^{\mathrm{Ch}}$ is linearization unstable at $g_{\mathrm{o}}$.

In particular, this theorem applies to the Kähler-Einstein metrics on compact Fano manifolds admitting an isometric Lie group action. On the other hand, we do not have any information on the general class of first-Chern-Einstein metrics with $\frac{1}{m} \mathrm{scal}^{\mathrm{Ch}}-\mathrm{d}^{*} \vartheta \geq 0$ at some point, which includes Ricci-flat Kähler metrics and Kähler-Einstein metrics with positive scalar curvature without global non-trivial Killing vector fields.

The paper is organized as follows. In Sect. 2, we summarize some basic facts on Geometric Analysis, on Complex Linear Algebra and on the Chern connection. In Sect. 3, we compute the first and second variation formulas for the Chern-scalar curvature, proving Proposition 3.6 and Proposition 3.10. In Sect. 4, we prove the main results Theorem A, Theorem B and Theorem C.

\section{Preliminaries and notation}

In this section, we summarize some basic facts on geometric analysis on Riemannian manifolds, referring to e.g. $[5,9,19,28]$, and we set the notation and some preliminary results concerning the geometry of Hermitian metrics, see e.g. [16].

\subsection{Basics on geometric analysis}

\subsubsection{A consequence of the Implicit Function Theorem}

Let $X, Y$ be Banach spaces, $\mathcal{U} \subset X$ an open set and $L(X, Y)$ the Banach space of continuous linear maps $T: X \rightarrow Y$. A map $f: \mathcal{U} \rightarrow Y$ is said to be of class $\mathcal{C}^{1}$ if there exists a continuous map $\mathrm{d} f: \mathcal{U} \rightarrow L(X, Y)$ called differential of $f$ such that

$$
\mathrm{d} f(x)(v)=\lim _{t \rightarrow 0} \frac{1}{t}(f(x+t v)-f(x)) \quad \text { for any } x \in \mathcal{U}, v \in X .
$$

Let us consider the Banach space $L^{(k)}(X, Y), k \in \mathbb{N}$, given by

$$
\begin{aligned}
L^{(k)}(X, Y) & :=\{k \text {-multilinear continuous maps } T: \underbrace{X \times \ldots \times X}_{k \text {-times }} \rightarrow Y\}, \\
\|T\|_{L^{(k)}(X, Y)} & :=\sup \left\{\left|T\left(x_{1}, \ldots, x_{k}\right)\right|_{Y}: x_{1}, \ldots, x_{k} \in X,\left|x_{1}\right|_{X}=\ldots=\left|x_{k}\right|_{X}=1\right\} .
\end{aligned}
$$

It can be directly checked that $L\left(X, L^{(k-1)}(X, Y)\right) \simeq L^{(k)}(X, Y)$ for any $k \in \mathbb{N}$. Therefore, this allows to give the following recursive definition: a map $f: \mathcal{U} \rightarrow Y$ is said to be of class $\mathcal{C}^{k}$ if it is of class $\mathcal{C}^{k-1}$ and there exists a continuous map $\mathrm{d}^{(k)} f: \mathcal{U} \rightarrow L^{(k)}(X, Y)$ such that $\mathrm{d}^{(k)} f(x)=\mathrm{d}\left(\mathrm{d}^{(k-1)} f\right)(x)$ for any $x \in \mathcal{U}$. As usual, $f$ is said to be smooth if 
it is of class $\mathcal{C}^{k}$ for any $k \in \mathbb{N}$. For the sake of shortness, we set $f_{x}^{\prime}(v):=\mathrm{d} f(x)(v)$ and $f_{x}^{\prime \prime}(v):=\mathrm{d}^{(2)} f(x)(v)$.

Let now $f: \mathcal{U} \rightarrow Y$ be a smooth map and $x_{\mathrm{o}} \in \mathcal{U}$ a point. We recall that $f$ is said to be a submersion at $x_{\mathrm{o}}$ if $f_{x_{0}}^{\prime}$ is surjective and the exact short sequence $\{0\} \rightarrow \operatorname{ker}\left(f_{x_{0}}^{\prime}\right) \rightarrow X \rightarrow$ $Y \rightarrow\{0\}$ splits, i.e. there exists a closed subspace $Z_{x_{0}} \subset X$ such that $X=\operatorname{ker}\left(f_{x_{0}}^{\prime}\right) \oplus Z_{x_{0}}$ and the restriction $f_{x_{0}}^{\prime} \mid Z_{x_{0}}$ is an isomorphism of Banach spaces from $Z_{x_{0}}$ to $Y$. By the Implicit Function Theorem, see e.g. [5, p. 72], if $f$ is submersion at $x_{0}$, it follows that:

i) $f$ is locally surjective at $x_{\mathrm{o}}$, i.e. $f$ maps any neighborhood of $x_{\mathrm{o}}$ in $X$ onto a neighborhood of $f\left(x_{\mathrm{o}}\right)$ in $Y$;

ii) the preimage $S_{f\left(x_{0}\right)}:=f^{-1}\left(f\left(x_{0}\right)\right)$ is a smooth submanifold of $X$ in a neighborhood of $x_{\mathrm{o}}$ with tangent space $T_{x_{\mathrm{o}}} S=\operatorname{ker}\left(f_{x_{\mathrm{o}}}^{\prime}\right)$.

Finally, we recall that $f: \mathcal{U} \rightarrow Y$ is said to be linearization stable at $x_{0}$ if, for any $v \in$ $\operatorname{ker}\left(f_{x_{0}}^{\prime}\right) \subset X$, there exists a smooth path $x:(-\epsilon, \epsilon) \rightarrow X$ such that $x(0)=x_{0}, \dot{x}(0)=v$ and $f(x(t))=f\left(x_{\mathrm{o}}\right)$ for any $-\epsilon<t<\epsilon$, see [12, p. 519]. Notice that, if $f$ is submersion at $x_{\mathrm{o}}$, then it is also linearization stable at $x_{\mathrm{o}}$, but the converse assertion is not true.

\subsubsection{Sobolev spaces on Riemannian manifolds}

Let $M$ be a connected, compact, oriented smooth manifold without boundary of even dimension $\operatorname{dim} M=2 m, g$ a fixed background Riemannian metric on $M$ and $v_{g}$ the induced Riemannian volume form. We extend $g$ to a Riemannian metric on the fibers of the bundle $\mathcal{T}^{(r, s)} M \rightarrow M$ of $(r, s)$-tensors over $M$ in the usual way and we denote by $D^{g}: \mathcal{C}^{\infty}\left(M, \mathcal{T}^{(r, s)} M\right) \rightarrow \mathcal{C}^{\infty}\left(M, \mathcal{T}^{(r, s+1)} M\right)$ the Levi-Civita connection of $g$.

Let $E \rightarrow M$ be any vector subbundle of $\mathcal{T}^{(\cdot, \cdot)} M$. For any integer $k \geq 0$ and for any tensor fields $h_{1}, h_{2} \in \mathcal{C}^{\infty}(M, E)$, we define the bilinear form

$$
\left\langle h_{1}, h_{2}\right\rangle_{W^{k, 2}}:=\sum_{0 \leq i \leq k} \int_{M} g\left(\left(D^{g}\right)^{i} h_{1},\left(D^{g}\right)^{i} h_{2}\right) v_{g} .
$$

Since $M$ is compact, the topology induced by the norm $\|\cdot\|_{W^{k, 2}}:=\sqrt{\langle\cdot, \cdot\rangle_{W^{k, 2}}}$ is independent of the Riemannian metric $g$ (see e.g. [19, Prop 2.2]). Accordingly, the Sobolev space $W^{k, 2}(M, E)$ is defined as the completion of $\mathcal{C}^{\infty}(M, E)$ with respect to the norm $\|\cdot\|_{W^{k, 2}}$. For the sake of notation, we set $L^{2}:=W^{0,2}$.

Notice that $\left(W^{k, 2}(M, E),\langle,\rangle_{W^{k, 2}}\right)$ is a Hilbert space. Moreover, for any $k \geq m+1$, by the Sobolev Embeddings Theorem (see e.g. [19, Thm 2.7]) there exists a continuous embedding

$$
\left(W^{k, 2}(M, E),\|\cdot\|_{W^{k, 2}}\right) \hookrightarrow\left(\mathcal{C}^{k-m-1}(M, E),\|\cdot\|_{\mathcal{C}^{k-m-1}}\right),
$$

where $\|\cdot\|_{\mathcal{C}^{k^{\prime}}}$ denotes the usual $\mathcal{C}^{k^{\prime}}$-norm for any integer $k^{\prime} \geq 0$.

Remark 2.1 We stress that (2.1) implies that $W^{k, 2}(M, E)$ consists of continuous sections if $k \geq m+1$. In particular, it is possible to define $W^{k, 2}(M, E)$ with $k \geq m+1$ for any subbundle $E$ of $\mathcal{T}^{(\cdot, \cdot)} M$. A remarkable example is the space of Riemannian metrics of class $W^{k, 2}$ defined as

$$
\mathcal{M}^{k}:=W^{k, 2}\left(M, S_{+}^{2}\left(T^{*} M\right)\right) .
$$

Let now $E, F$ be two vector subbundle of $\mathcal{T}^{(\cdot, \cdot \cdot)} M$ and $P: \mathcal{C}^{\infty}(M, E) \rightarrow \mathcal{C}^{\infty}(M, F)$ a linear differential operator of order $r$. We recall that: 
- the principal symbol of $P$ is the endomorphism $\sigma(P): T^{*} M \otimes E \rightarrow F$ defined as follows: for any $x \in M, \xi \in T_{x}^{*} M, s \in E_{x}$

$$
\sigma(P)_{x}(\xi \otimes s):=\frac{1}{r !} P\left(\phi^{r} u\right)(x),
$$

where $\phi \in \mathcal{C}^{\infty}(M, \mathbb{R})$ verifies $\phi(x)=0, \mathrm{~d} \phi_{x}=\xi$ and $u \in \mathcal{C}^{\infty}(M, E)$ verifies $u(x)=s$;

- the $L^{2}$-adjoint of $P$ is the unique linear differential operator $P^{*}: \mathcal{C}^{\infty}(M, F) \rightarrow$ $\mathcal{C}^{\infty}(M, E)$ of order $r$ satisfying

$$
\left\langle P\left(s_{1}\right), s_{2}\right\rangle_{L^{2}}=\left\langle s_{1}, P^{*}\left(s_{2}\right)\right\rangle_{L^{2}} \text { for any } s_{1} \in \mathcal{C}^{\infty}(M, E), s_{2} \in \mathcal{C}^{\infty}(M, F) .
$$

We also remark that $P$ can be uniquely extended to a linear differential operator $P$ : $W^{k+r, 2}(M, E) \rightarrow W^{k, 2}(M, F)$ for any integer $k \geq 0$ (see [28, Thm 6, p. 152]). Then, we have the following

Theorem 2.2 (Berger-Ebin Splitting Lemma [7, Thm 4.1]) Let $P: \mathcal{C}^{\infty}(M, E) \rightarrow \mathcal{C}^{\infty}(M, F)$ be a linear differential operator of order $r$ and $k \in \mathbb{N}$ such that $k \geq r$. If $P$ has injective symbol or its $L^{2}$-adjoint $P^{*}$ has injective symbol, then

$$
W^{k, 2}(M, F)=\operatorname{Im}(P) \oplus \operatorname{ker}\left(P^{*}\right),
$$

where $P$ is extended to $P: W^{k+r, 2}(M, E) \rightarrow W^{k, 2}(M, F)$ and, consequently, $P^{*}$ : $W^{k, 2}(M, F) \rightarrow W^{k-r, 2}(M, E)$. Moreover, if $P$ has injective symbol, then $\operatorname{ker}(P) \subset$ $W^{k+r, 2}(M, E)$ is finite dimensional and consists of smooth sections.

Remark 2.3 As a consequence of the Berger-Ebin Splitting Lemma, we remark that: if $P^{*}$ is injective and has injective symbol, then $P$ is surjective and its kernel splits.

\subsection{Complex linear algebra}

Let $V=(V, J, g)$ be a triple given by a real vector space $V$ of dimension $\operatorname{dim}_{\mathbb{R}} V=2 m$, a linear complex structure $J$ on $V$ and an Euclidean scalar product $g$ on $V$ such that $g(J(\cdot), J(\cdot \cdot))=g(\cdot, \cdot \cdot)$. The complexification $V^{\mathbb{C}}:=V \otimes_{\mathbb{R}} \mathbb{C}$ splits as a sum of $J-$ eigenspaces $V^{\mathbb{C}}=V^{1,0} \oplus V^{0,1}$ and all the real tensors on $V$ can be uniquely $\mathbb{C}$-linearly extended to $V^{\mathbb{C}}$. Fix a $(J, g)$-unitary basis $\left(e_{i}, J e_{i}\right)$ for $V$, and consider the associated complex basis

$$
\varepsilon_{i}:=\frac{1}{\sqrt{2}}\left(e_{i}-i J e_{i}\right), \quad \varepsilon_{i}^{-}:=\frac{1}{\sqrt{2}}\left(e_{i}+i J e_{i}\right)
$$

for $V^{\mathbb{C}}$, which is unitary with respect to the Hermitian extension of $g$ to $V^{\mathbb{C}}$. Clearly, it holds that $\overline{\varepsilon_{i}}=\varepsilon_{\bar{i}}^{-}$and $J \varepsilon_{i}=i \varepsilon_{i}, J \varepsilon_{\bar{i}}^{-}=-i \varepsilon_{\bar{i}}^{-}$. Moreover, $J$ acts on covectors $\vartheta \in V^{*}$ via $(J \vartheta):=-\vartheta \circ J$, so that $\left(e^{i}, J e^{i}\right)$ is the dual basis of $\left(e_{i}, J e_{i}\right)$ for $V^{*}$. For the complexification, we get that

$$
\varepsilon^{i}:=\frac{1}{\sqrt{2}}\left(e^{i}+i J e^{i}\right), \quad \varepsilon^{\bar{i}}:=\frac{1}{\sqrt{2}}\left(e_{i}-i J e_{i}\right)
$$

is the dual basis of $\left(\varepsilon_{i}, \varepsilon_{\bar{i}}\right)$, and $J \varepsilon^{i}=-i \varepsilon^{i}, J \varepsilon^{\bar{i}}=i \varepsilon^{\bar{i}}$. With respect to such basis, we have

$$
g=\delta_{\bar{j} i} \varepsilon^{i} \odot \varepsilon^{\bar{j}}, \quad \text { with } \varepsilon^{i} \odot \varepsilon^{\bar{j}}:=\varepsilon^{i} \otimes \varepsilon^{\bar{j}}+\varepsilon^{\bar{j}} \otimes \varepsilon^{i} .
$$

We consider now the spaces

$$
\begin{aligned}
\operatorname{Sym}^{1,1}(V) & :=\{h \in \operatorname{End}(V): g(h(\cdot), \cdot \cdot)=g(\cdot, h(\cdot \cdot)),[h, J]=0\}, \\
\operatorname{Skew}^{1,1}(V) & :=\{\tilde{h} \in \operatorname{End}(V): g(\tilde{h}(\cdot), \cdot \cdot)=-g(\cdot, \tilde{h}(\cdot \cdot)),[\tilde{h}, J]=0\} .
\end{aligned}
$$


Notice now that any $h \in \operatorname{Sym}^{1,1}(V)$ preserves the decomposition $V^{\mathbb{C}}=V^{1,0} \oplus V^{0,1}$ and takes the form

$$
h=h_{i}^{j} \varepsilon_{j} \otimes \varepsilon^{i}+h_{\bar{i}}^{\bar{j}} \varepsilon_{\bar{j}} \otimes \varepsilon^{\bar{i}}, \quad \text { with } h_{i}^{j} \in \mathbb{C} \text { and } h_{\bar{i}}^{\bar{j}}=\overline{h_{i}^{j}}=h_{j}^{i} .
$$

Moreover, the linear map

$$
\operatorname{Sym}^{1,1}(V) \rightarrow \operatorname{Skew}^{1,1}(V), \quad h \mapsto \tilde{h}=J \circ h
$$

is an isomorphism, with inverse given by $\tilde{h} \mapsto h=-J \circ \tilde{h}$. Then, we denote by $\operatorname{tr}^{\mathbb{R}}$ : $\operatorname{Sym}^{1,1}(V) \rightarrow \mathbb{R}$ the trace of the real endomorphism $h: V \rightarrow V$ and by $\operatorname{tr}^{\mathbb{C}}: \operatorname{Sym}^{1,1}(V) \rightarrow$ $\mathbb{R}$ the trace of the complex endomorphism $h: V^{1,0} \rightarrow V^{1,0}$, which are related by

$$
\begin{aligned}
\operatorname{tr}^{\mathbb{R}}(h) & =\sum_{1 \leq i \leq m}\left(g\left(h\left(e_{i}\right), e_{i}\right)+g\left(h\left(J e_{i}\right), J e_{i}\right)\right) \\
& =2 \sum_{1 \leq i \leq m} g\left(h\left(\varepsilon_{i}\right), \varepsilon_{\bar{i}}^{-}\right)=2 \operatorname{tr}^{\mathbb{C}}(h) .
\end{aligned}
$$

Finally, we consider the space

$$
\Lambda^{1,1}\left(V^{*}\right):=\left\{\alpha \in \Lambda^{2}\left(V^{*}\right): a(J(\cdot), J(\cdot \cdot))=\alpha(\cdot, \cdot \cdot)\right\},
$$

and we observe that the linear map

$$
\rho_{g}: \operatorname{Sym}^{1,1}(V) \rightarrow \Lambda^{1,1}\left(V^{*}\right), \quad \rho_{g}(h):=g((J \circ h) \cdot, \cdot \cdot)
$$

is an isomorphism. Accordingly, we define

$$
\operatorname{Tr}_{g}^{\mathbb{C}}: \Lambda^{1,1}\left(V^{*}\right) \rightarrow \mathbb{R}, \quad \operatorname{Tr}_{g}^{\mathbb{C}}(\alpha):=\operatorname{tr}^{\mathbb{C}}\left(\rho_{g}^{-1}(\alpha)\right) .
$$

Since any $\alpha \in \Lambda^{1,1}\left(V^{*}\right)$ is of the form

$$
\alpha=\alpha_{\bar{j} i} i \varepsilon^{i} \wedge \varepsilon^{\bar{j}}, \quad \text { with } \varepsilon^{i} \wedge \varepsilon^{\bar{j}}:=\varepsilon^{i} \otimes \varepsilon^{\bar{j}}-\varepsilon^{\bar{j}} \otimes \varepsilon^{i},
$$

an easy computation shows that

$$
\operatorname{Tr}_{g}^{\mathbb{C}}(\alpha)=\sum_{1 \leq i \leq m} \alpha\left(e_{i}, J e_{i}\right)=-i \sum_{1 \leq i \leq m} \alpha\left(\varepsilon_{i}, \varepsilon_{\bar{i}}\right)=\delta^{i \bar{j}} \alpha_{\bar{j} i} .
$$

\subsection{The Chern connection}

Let $(M, J, g)$ be a compact, connected, Hermitian manifold of dimension $\operatorname{dim}_{\mathbb{R}} M=2 m$ and let $\omega:=\rho_{g}(\mathrm{Id})=g(J \cdot, \cdot)$ be its fundamental 2-form, where $\operatorname{Id} \in \mathcal{C}^{\infty}\left(M, \operatorname{Sym}^{1,1}(T M)\right)$ is the identity endomorphism, $D^{g}$ its Levi-Civita connection and $\nabla$ its Chern connection, defined by

$$
g\left(\nabla_{X} Y, Z\right):=g\left(D_{X}^{g} Y, Z\right)-\frac{1}{2} \mathrm{~d} \omega(J X, Y, Z)
$$

for any $X, Y, Z \in \mathcal{C}^{\infty}(M, T M)$. It is well-known that the Chern connection is characterized by the following properties, see e.g. [16, p. 273]:

$$
\nabla g=0, \quad \nabla J=0, \quad J(T(X, Y))=T(J X, Y)=T(X, J Y),
$$

where $T(X, Y):=\nabla_{X} Y-\nabla_{Y} X-[X, Y]$ is the torsion tensor of $\nabla$, which can be expressed by (see e.g. [16, Prop 4])

$$
-2 g(T(X, Y), Z)=\mathrm{d} \omega(J X, Y, Z)+\mathrm{d} \omega(X, J Y, Z) .
$$


We denote by

$$
\left.\vartheta(X):=\operatorname{tr}^{\mathbb{R}}(T(X, \cdot))=\operatorname{Tr}_{g}^{\mathbb{C}}(X\lrcorner \mathrm{d} \omega\right)
$$

the Lee form. We also define the Chern-curvature operator by

$$
\Omega(g) \in \mathcal{C}^{\infty}\left(M, \Lambda^{1,1}\left(T^{*} M\right) \otimes \operatorname{Skew}^{1,1}(T M)\right), \quad \Omega(g)(X, Y):=\nabla_{[X, Y]}-\left[\nabla_{X}, \nabla_{Y}\right] .
$$

Moreover, we call first Chern-Ricci form the tensor field

$$
\tilde{S}(g) \in \mathcal{C}^{\infty}\left(M, \Lambda^{1,1}\left(T^{*} M\right)\right), \quad \tilde{S}(g)(X, Y):=-\operatorname{tr}^{\mathbb{C}}(J \circ \Omega(g)(X, Y))
$$

and first Chern-Ricci symmetric endomorphism

$$
S(g) \in \mathcal{C}^{\infty}\left(M, \operatorname{Sym}^{1,1}(T M)\right), \quad S(g):=\rho_{g}^{-1}(\tilde{S}(g)) .
$$

Finally, the Chern-scalar curvature is the trace

$$
\operatorname{scal}^{\mathrm{Ch}}(g) \in \mathcal{C}^{\infty}(M, \mathbb{R}), \quad \operatorname{scal}^{\mathrm{Ch}}(g):=2 \operatorname{Tr}_{g}^{\mathbb{C}}(\tilde{S}(g)) .
$$

We remark that, with this notation, when $g$ is Kähler it holds that

$$
S(g)=\operatorname{Ric}(g), \quad \operatorname{scal}^{\mathrm{Ch}}(g)=\operatorname{scal}(g),
$$

where Ric $(g)$ and scal $(g)$ denote the Riemannian Ricci endomorphism and the Riemannian scalar curvature of $g$, respectively. We recall that $g$ is called first-Chern-Einstein if it satisfies $S(g)=\frac{\lambda}{2 m} \operatorname{Id}$ for some $\lambda \in \mathcal{C}^{\infty}(M, \mathbb{R})$, see [3,31] and references therein. Notice that, in this case $\lambda=\operatorname{scal}^{\mathrm{Ch}}(g)$ and, if $g$ is Kähler, then this notion corresponds to the Kähler-Einstein condition.

We also set $\mathrm{d}^{c}:=J^{-1} \circ \mathrm{d} \circ J$, so that

$$
\mathrm{d}=\partial+\bar{\partial}, \quad \mathrm{d}^{c}=-i(\partial-\bar{\partial}), \quad \mathrm{dd}^{c}=2 \mathrm{i} \partial \bar{\partial}
$$

and we denote by $\Delta_{g}:=\left(D^{g}\right)^{*} D^{g}$ the Laplace-Beltrami operator. We recall that $\mathrm{dd}^{c}$ and $\Delta_{g}$ are related by the following

Lemma 2.4 (see e.g. $\left[15\right.$, p. 502]) For any function $u \in \mathcal{C}^{\infty}(M, \mathbb{R})$ it holds that

$$
\operatorname{Tr}_{g}^{\mathbb{C}}\left(\operatorname{dd}^{c} u\right)=\Delta_{g} u+g(\mathrm{~d} u, \vartheta) .
$$

Proof For the sake of completeness, we summarize here the computation. By the very definition, for any vector fields $X, Y$ it holds that

$$
\operatorname{dd}^{c} u(X, Y)=\mathcal{L}_{X} \mathcal{L}_{J Y} u-\mathcal{L}_{Y} \mathcal{L}_{J X} u-\mathcal{L}_{J[X, Y]} u .
$$

Let now $\left(\tilde{e}_{\alpha}\right)=\left(e_{i}, J e_{i}\right)$ be a local $(J, g)$-unitary frame on $M$ and set $A:=\nabla-D^{g}$. Then, notice that

$$
\begin{aligned}
\sum_{1 \leq i \leq m}-J\left[e_{i}, J e_{i}\right] & =\sum_{1 \leq i \leq m}-J\left(\nabla_{e_{i}} J e_{i}-\nabla_{J e_{i}} e_{i}-T\left(e_{i}, J e_{i}\right)\right) \\
& =\sum_{1 \leq \alpha \leq 2 m} \nabla_{\tilde{e}_{\alpha}} \tilde{e}_{\alpha}
\end{aligned}
$$


and so

$$
\begin{aligned}
\operatorname{Tr}_{g}^{\mathbb{C}}\left(\operatorname{dd}^{c} u\right) & =\sum_{1 \leq i \leq m}\left(\operatorname{dd}^{c} u\right)\left(e_{i}, J e_{i}\right) \\
& =-\sum_{1 \leq i \leq m}\left(\mathcal{L}_{e_{i}} \mathcal{L}_{e_{i}} u+\mathcal{L}_{J e_{i}} \mathcal{L}_{J e_{i}} u\right)-\sum_{1 \leq i \leq m} \mathcal{L}_{J\left[e_{i}, J e_{i}\right]} u \\
& =\sum_{1 \leq \alpha \leq 2 m}-\left(\mathcal{L}_{\tilde{e}_{\alpha}} \mathcal{L}_{\tilde{e}_{\alpha}}-\mathcal{L}_{D_{\tilde{e}_{\alpha}}^{g} \tilde{e}_{\alpha}}\right) u+\sum_{1 \leq \alpha \leq 2 m} \mathcal{L}_{A\left(\tilde{e}_{\alpha}, \tilde{e}_{\alpha}\right)} u .
\end{aligned}
$$

Moreover, from (2.3) we get

$$
\begin{aligned}
\sum_{1 \leq \alpha \leq 2 m} A\left(\tilde{e}_{\alpha}, \tilde{e}_{\alpha}\right) & =\sum_{1 \leq \alpha, \beta \leq 2 m}-\frac{1}{2} \mathrm{~d} \omega\left(J \tilde{e}_{\alpha}, \tilde{e}_{\alpha}, \tilde{e}_{\beta}\right) \tilde{e}_{\beta} \\
& =\sum_{1 \leq \beta \leq 2 m} \sum_{1 \leq i \leq m} \mathrm{~d} \omega\left(\tilde{e}_{\beta}, e_{i}, J e_{i}\right) \tilde{e}_{\beta}=\sum_{1 \leq \beta \leq 2 m} \vartheta\left(\tilde{e}_{\beta}\right) \tilde{e}_{\beta}=\vartheta^{\#}
\end{aligned}
$$

and therefore we obtain (2.8).

Given a function $u \in \mathcal{C}^{\infty}(M, \mathbb{R})$, we also denote by $\operatorname{Hess}_{g}(u) \in \mathcal{C}^{\infty}(M, \operatorname{Sym}(T M))$ the Hessian of $u$ defined by

$$
g\left(\operatorname{Hess}_{g}(u)(X), Y\right):=\left(D_{X}^{g}(\mathrm{~d} u)\right)(Y)
$$

and we stress the following

Lemma 2.5 If $g$ is Kähler, then

$$
\operatorname{dd}^{c} u(X, Y)=g\left(\operatorname{Hess}_{g}(u)(X), J Y\right)-g\left(\operatorname{Hess}_{g}(u)(J X), Y\right) .
$$

Proof By the very definition, we have

$$
g\left(\operatorname{Hess}_{g}(u)(X), Y\right)=\mathcal{L}_{X} \mathcal{L}_{Y} u-\mathcal{L}_{D_{X}^{g} Y} u .
$$

Therefore, since $g$ is Kähler, we get

$$
\begin{aligned}
g & \left(\operatorname{Hess}_{g}(u)(X), J Y\right)-g\left(\operatorname{Hess}_{g}(u)(J X), Y\right) \\
= & \mathcal{L}_{X} \mathcal{L}_{J Y} u-\mathcal{L}_{D_{X}^{g}(J Y)} u-\mathcal{L}_{J X} \mathcal{L}_{Y} u+\mathcal{L}_{D_{J X}^{g} Y} u \\
= & \mathcal{L}_{X} \mathcal{L}_{J Y} u-\mathcal{L}_{Y} \mathcal{L}_{J X} u-\mathcal{L}_{[J X, Y]} u-\mathcal{L}_{J D_{X}^{g} Y} u \\
& +\mathcal{L}_{J D_{Y}^{g} X} u+\mathcal{L}_{[J X, Y]} u \\
= & \mathcal{L}_{X} \mathcal{L}_{J Y} u-\mathcal{L}_{Y} \mathcal{L}_{J X} u-\mathcal{L}_{J[X, Y]} u \\
= & \operatorname{dd}^{c} u(X, Y)
\end{aligned}
$$

which concludes the proof.

Finally, we introduce the following two operators

$$
\delta_{g}, \delta^{\nabla}: \mathcal{C}^{\infty}(M, \operatorname{Sym}(T M)) \rightarrow \mathcal{C}^{\infty}\left(M, T^{*} M\right)
$$

defined by

$$
\left(\delta_{g} h\right)(X):=\operatorname{tr}^{\mathbb{R}}\left(\left(D^{g} h\right) X\right), \quad\left(\delta^{\nabla} h\right)(X):=\operatorname{tr}^{\mathbb{R}}((\nabla h) X)
$$




\section{Variation formulas for the Chern-scalar curvature}

Let $(M, J)$ be a compact, connected, complex manifold of dimension $\operatorname{dim}_{\mathbb{R}} M=2 \mathrm{~m}$. We consider the bundle $S_{+}^{1,1}\left(T^{*} M\right) \rightarrow M$ of symmetric, bilinear, positive definite, $J$-invariant forms and, for any integer $k \geq m+1$, we define the space of Hermitian metrics on $(M, J)$ of class $W^{2, k}$ as

$$
\mathcal{M}_{\mathrm{H}}^{k}:=W^{k, 2}\left(M, S_{+}^{1,1}\left(T^{*} M\right)\right)
$$

For the sake of notation, we denote the space of smooth Hermitian metrics by $\mathcal{M}_{\mathrm{H}}:=$ $\mathcal{C}^{\infty}\left(M, S_{+}^{1,1}\left(T^{*} M\right)\right)$.

\subsection{First variation of the Chern-scalar curvature}

Fix a smooth Hermitian metric $g \in \mathcal{M}_{\mathrm{H}}$. Then, given an element $h \in \mathcal{C}^{\infty}\left(M, \operatorname{Sym}^{1,1}\right.$ $(T M)$ ), we consider the corresponding path $\left(g_{t}\right) \subset \mathcal{M}_{\mathrm{H}}$ given by

$$
g_{t}:=g((\operatorname{Id}+t h) \cdot, \cdot \cdot), \quad t \in(-\epsilon, \epsilon)
$$

with $\epsilon>0$ small enough. For the sake of notation, in this section we will always use this shortener notation: if $F$ is a function defined on $\mathcal{M}_{\mathrm{H}}$, we write $F^{\prime}$ instead of $F_{g}^{\prime}(h)$ to denote the differential of $F$ at $g$ in the direction of $h$.

Proposition 3.1 The differential at $g$ in the direction of $h$ of the Chern connection is the $(1,2)$-tensor field $\nabla^{\prime}$ defined by

$$
2 \nabla_{X}^{\prime} Y=\left(\nabla_{X} h\right)(Y)-\left(J \circ \nabla_{J X} h\right)(Y)
$$

Proof We set $C^{t}:=\nabla^{g_{t}}-\nabla^{g}$, that is a $(2,1)$-tensor field. Then, by using the Koszul Formula of the Chern connection (see e.g. [4, Sect 2.1])

$$
\begin{aligned}
2 g_{t}\left(C_{X}^{t} Y, Z\right)= & 2 g_{t}\left(\nabla_{X}^{g_{t}} Y, Z\right)-2 g_{t}\left(\nabla_{X}^{g} Y, Z\right) \\
= & \mathcal{L}_{X}\left(g_{t}(Y, Z)\right)-\mathcal{L}_{X}(g(Y, Z))-\mathcal{L}_{J X}\left(g_{t}(J Y, Z)\right)+\mathcal{L}_{J X}(g(J Y, Z)) \\
& +g_{t}([X, Y], Z)-g([X, Y], Z)-g_{t}([J X, Y], J Z)+g([J X, Y], J Z) \\
& -g_{t}([X, Z], Y)+g([X, Z], Y)+g_{t}([J X, Z], J Y) \\
& -g([J X, Z], J Y)-2 \operatorname{tg}\left(h\left(\nabla_{X}^{g} Y\right), Z\right) \\
= & t \mathcal{L}_{X}(g(h(Y), Z))-t \mathcal{L}_{J X}(g(h(J Y), Z))+\operatorname{tg}(h([X, Y]), Z) \\
& -\operatorname{tg}(h([J X, Y]), J Z)-\operatorname{tg}(h([X, Z]), Y) \\
& +\operatorname{tg}(h([J X, Z]), J Y)-2 \operatorname{tg}\left(h\left(\nabla_{X}^{g} Y\right), Z\right)
\end{aligned}
$$


and so

$$
\begin{aligned}
& 2 g\left(\left(\frac{1}{t} C^{t}\right)_{X} Y, Z\right)+2 g\left(h\left(C_{X}^{t} Y\right), Z\right)= \\
&= g\left(\left(\nabla_{X}^{g} h\right)(Y), Z\right)-g\left(\left(\nabla_{J X}^{g} h\right)(J Y), Z\right)-g\left(h\left(\nabla_{X}^{g} Y\right), Z\right) \\
&+g(h([X, Y]), Z)+g\left(h\left(\nabla_{X}^{g} Z\right), Y\right) \\
& \quad-g(h([X, Z]), Y)+g\left(h\left(\nabla_{J X}^{g} Y\right), J Z\right)-g(h([J X, Y]), J Z) \\
&-g\left(h\left(\nabla_{J X}^{g} Z\right), J Y\right)+g(h([J X, Z]), J Y) \\
&= g\left(\left(\nabla_{X}^{g} h\right)(Y), Z\right)-g\left(\left(\nabla_{J X}^{g} h\right)(J Y), Z\right)-g\left(h\left(\nabla_{Y}^{g} X+T^{g}(X, Y)\right), Z\right) \\
& \quad+g\left(h\left(\nabla_{Y}^{g} X+T^{g}(X, Y)\right), Z\right) \\
& \quad+g\left(h\left(\nabla_{Z}^{g} X+T^{g}(X, Z)\right), Y\right)-g\left(h\left(\nabla_{Z}^{g} X+T^{g}(X, Z)\right), Y\right) \\
&= g\left(\left(\nabla_{X}^{g} h\right)(Y), Z\right)-g\left(\left(\nabla_{J X}^{g} h\right)(J Y), Z\right) .
\end{aligned}
$$

Letting $t \rightarrow 0$, we obtain (3.1).

Lemma 3.2 The differential at $g$ in the direction of $h$ of the complex trace is the linear map defined, for $\alpha \in \mathcal{C}^{\infty}\left(M, \Lambda^{1,1}\left(T^{*} M\right)\right)$, by

$$
\left(\operatorname{Tr}^{\mathbb{C}}\right)^{\prime}(\alpha)=-\frac{1}{2} g\left(h, \rho_{g}^{-1}(\alpha)\right) .
$$

Proof Fix a local $(J, g)$-unitary frame $\left(e_{i}, J e_{i}\right)$ on $M$ and the associated frame

$$
\varepsilon_{i}:=\frac{1}{\sqrt{2}}\left(e_{i}-i J e_{i}\right), \quad \varepsilon_{i}^{-}:=\frac{1}{\sqrt{2}}\left(e_{i}+i J e_{i}\right),
$$

so that

$$
g=\delta_{\bar{j} i} \varepsilon^{i} \odot \varepsilon^{\bar{j}}, \quad \rho_{g}(h)=h_{\bar{j} i} i \varepsilon^{i} \wedge \varepsilon^{\bar{j}}, \quad \alpha=\alpha_{\bar{j} i} i \varepsilon^{i} \wedge \varepsilon^{\bar{j}},
$$

where $h_{\bar{j} i}=\delta_{\bar{j} s} h_{i}^{s}$. If $\left(g_{t}\right)_{\bar{j} i}:=\delta_{\bar{j} i}+t h_{\bar{j} i}$ and $\left(\left(g_{t}\right)^{i \bar{j}}\right):=\left(\left(g_{t}\right)_{\bar{j} i}\right)^{-1}$, it follows that

$$
\left(g_{t}\right)^{i \bar{j}}=\delta^{i \bar{j}}-\operatorname{tg}^{i \bar{r}} h_{\bar{r} s}\left(g_{t}\right)^{s \bar{j}} .
$$

Since $\operatorname{Tr}_{g}^{\mathbb{C}}(\alpha)=\delta^{i} \bar{j}_{\alpha \bar{j} i}$, we get

$$
\begin{aligned}
\left(\operatorname{Tr}^{\mathbb{C}}\right)^{\prime}(\alpha) & =\lim _{t \rightarrow 0} \frac{1}{t}\left(\operatorname{Tr}_{g_{t}}^{\mathbb{C}}(\alpha)-\operatorname{Tr}_{g}^{\mathbb{C}}(\alpha)\right) \\
& =\lim _{t \rightarrow 0} \frac{1}{t}\left(\left(g_{t}\right)^{i \bar{j}}-\delta^{i \bar{j}}\right) \alpha_{\bar{j} i}=-h^{i \bar{j}} \alpha_{\bar{j} i},
\end{aligned}
$$

where $h^{i} \bar{j}=h_{s}^{i} \delta^{s \bar{j}}$. On the other hand, one can directly check that

$$
g\left(h, \rho_{g}^{-1}(\alpha)\right)=2 h^{i} \bar{j}_{\bar{j} i},
$$

which completes the proof.

Proposition 3.3 The differential at $g$ in the direction of $h$ of the Chern-curature operator is given by

$$
2 \Omega^{\prime}(X, Y)=[\Omega(X, Y), h]+J \circ\left(\nabla_{X} \nabla_{J Y} h-\nabla_{Y} \nabla_{J X} h-\nabla_{J[X, Y]} h\right) .
$$

Proof By differentiating (2.5), we get

$$
\Omega^{\prime}(X, Y)=\nabla_{[X, Y]}^{\prime}-\left[\nabla_{X}, \nabla_{Y}^{\prime}\right]-\left[\nabla_{X}^{\prime}, \nabla_{Y}\right] .
$$


Notice that

$$
2\left[\nabla_{X}, \nabla_{Y}^{\prime}\right]=\nabla_{X} \nabla_{Y} h-J \circ \nabla_{X} \nabla_{J Y} h
$$

and so we get

$$
\begin{aligned}
2 \Omega^{\prime}(X, Y)= & \nabla_{[X, Y]} h-J \circ \nabla_{J[X, Y]} h-\nabla_{X} \nabla_{Y} h+J \circ \nabla_{X} \nabla_{J Y} h \\
& +\nabla_{Y} \nabla_{X} h-J \circ \nabla_{Y} \nabla_{J X} h \\
= & \Omega_{X, Y} h+J \circ\left(\nabla_{X} \nabla_{J Y} h-\nabla_{Y} \nabla_{J X} h-\nabla_{J[X, Y]} h\right) .
\end{aligned}
$$

Here, by a slight abuse of notation, we denoted by $\Omega_{X, Y}$ the curvature of the connection naturally induced on the bundle $\operatorname{End}(T M)$. Since $\Omega_{X, Y} h=[\Omega(X, Y), h]$, we obtain (3.3).

Lemma 3.4 For any vector field $X$ it holds that

$$
\operatorname{tr}^{\mathbb{C}}\left(\nabla_{X} h\right)=\mathcal{L}_{X}\left(\operatorname{tr}^{\mathbb{C}} h\right)
$$

Proof Fix $x \in M$ and let $\left(\tilde{e}_{\alpha}\right)$ be a local orthonormal frame around $x$ such that $\left(D \tilde{e}_{\alpha}\right)_{x}=0$. Then, by using (2.3), at the point $x$ we get

$$
\begin{aligned}
\operatorname{tr}^{\mathbb{R}}\left(\nabla_{X} h\right) & =\sum_{\alpha} g\left(\left(\nabla_{X} h\right)\left(\tilde{e}_{\alpha}\right), \tilde{e}_{\alpha}\right) \\
& =\sum_{\alpha} g\left(\nabla_{X}\left(h\left(\tilde{e}_{\alpha}\right)\right), \tilde{e}_{\alpha}\right)-g\left(h\left(\nabla_{X} \tilde{e}_{\alpha}\right), \tilde{e}_{\alpha}\right) \\
& =\sum_{\alpha} \mathcal{L}_{X} g\left(h\left(\tilde{e}_{\alpha}\right), \tilde{e}_{\alpha}\right)-2 g\left(h\left(\tilde{e}_{\alpha}\right), \nabla_{X} \tilde{e}_{\alpha}\right) \\
& =\mathcal{L}_{X}\left(\operatorname{tr}^{\mathbb{R}} h\right)+\sum_{\alpha, \beta} \mathrm{d} \omega\left(J X, \tilde{e}_{\alpha}, \tilde{e}_{\beta}\right) g\left(h\left(\tilde{e}_{\alpha}\right), \tilde{e}_{\beta}\right) \\
& =2 \mathcal{L}_{X}\left(\operatorname{tr}^{\mathbb{C}} h\right) .
\end{aligned}
$$

Moreover, since $\nabla_{X} h \in \mathcal{C}^{\infty}\left(M, \operatorname{Sym}^{1,1}(T M)\right)$, it holds that

$$
\operatorname{tr}^{\mathbb{R}}\left(\nabla_{X} h\right)=2 \operatorname{tr}^{\mathbb{C}}\left(\nabla_{X} h\right)
$$

and so we get the thesis.

Proposition 3.5 The differential at $g$ in the direction of $h$ of the first Chern-Ricci form is given by

$$
\tilde{S}^{\prime}=\frac{1}{2} \mathrm{dd}^{c}\left(\operatorname{tr}^{\mathbb{C}} h\right)
$$

Proof Notice that the trace $\operatorname{tr}^{\mathbb{C}}$ commutes with the differentiation. Moreover, it is straightforward to check that, for any vector fields $X, Y$ and $h \in \mathcal{C}^{\infty}\left(M, \operatorname{Sym}^{1,1}(T M)\right)$, the commutator $[\Omega(X, Y), h]$ is a section of $\operatorname{Sym}^{1,1}(T M)$, with zero trace. Therefore, $J \circ[\Omega(X, Y), h] \in$ $\mathcal{C}^{\infty}\left(M\right.$, Skew $\left.^{1,1}(T M)\right)$, and so, by means of (2.6), (3.3), (3.4), and (2.9), we obtain

$$
\begin{aligned}
\tilde{S}^{\prime}(X, Y) & =\frac{1}{2} \operatorname{tr}^{\mathbb{C}}\left(\nabla_{X} \nabla_{J Y} h-\nabla_{Y} \nabla_{J X} h-\nabla_{J[X, Y]} h\right) \\
& =\frac{1}{2}\left(\mathcal{L}_{X} \mathcal{L}_{J Y}\left(\operatorname{tr}^{\mathbb{C}} h\right)-\mathcal{L}_{Y} \mathcal{L}_{J X}\left(\operatorname{tr}^{\mathbb{C}} h\right)-\mathcal{L}_{J[X, Y]}\left(\operatorname{tr}^{\mathbb{C}} h\right)\right) \\
& =\frac{1}{2} \operatorname{dd}^{c}\left(\operatorname{tr}^{\mathbb{C}} h\right)(X, Y)
\end{aligned}
$$

and so we get (3.5). 
As a direct consequence of (3.2) and (3.5), and by (2.8), we get

Proposition 3.6 The differential at $g$ in the direction of $h$ of the Chern-scalar curvature is given by

$$
\left(\mathrm{scal}^{\mathrm{Ch}}\right)^{\prime}=\Delta_{g}\left(\operatorname{tr}^{\mathbb{C}} h\right)+g\left(\mathrm{~d}\left(\operatorname{tr}^{\mathbb{C}} h\right), \vartheta\right)-g(h, S(g)) .
$$

\subsection{Second variation of the Chern-scalar curvature}

We are going to compute the second variation of the Chern-scalar curvature at a fixed point $g \in \mathcal{M}_{\mathrm{H}}$. Let $g_{t}=g((\mathrm{Id}+t h) \cdot, \cdot$.$) as before. From [9], and by recalling the definition$ of $\delta_{g}$ in (2.11), we have

Proposition 3.7 (see [9, Prop 1.184]) The differential at $g$ in the direction of h of the LaplaceBeltrami operator is given by

$$
\Delta^{\prime} u=g\left(\operatorname{Hess}_{g}(u), h\right)+g\left(\mathrm{~d} u, \delta_{g} h\right)-g\left(\mathrm{~d} u, \mathrm{~d}\left(\operatorname{tr}^{\mathbb{C}} h\right)\right) .
$$

By means of a straightforward computation in local coordinates, one can show that, for any $\alpha, \beta \in \mathcal{C}^{\infty}\left(M, T^{*} M\right)$ and $A, B \in \mathcal{C}^{\infty}(M, \operatorname{End}(T M))$, we have

$$
g(\alpha, \beta)^{\prime}=-g(\alpha \circ h, \beta), \quad g(A, B)^{\prime}=0 .
$$

For the following, we recall that $\delta^{\nabla}$ has been defined in (2.11):

Proposition 3.8 The differential at $g$ in the direction of $h$ of the Lee form is given by

$$
\vartheta^{\prime}=\mathrm{d}\left(\operatorname{tr}^{\mathbb{C}} h\right)-\delta^{\nabla} h .
$$

Proof By the very definition of Lee form (2.4), we have

$$
\vartheta^{\prime}(X)=\operatorname{tr}^{\mathbb{R}}\left(T^{\prime}(X, \cdot)\right)=\operatorname{tr}^{\mathbb{R}}\left(\nabla_{X}^{\prime}(\cdot)\right)-\operatorname{tr}^{\mathbb{R}}\left(\nabla_{(\cdot)}^{\prime} X\right) .
$$

Then, from (3.1) and (3.4), it follows that

$$
\operatorname{tr}^{\mathbb{R}}\left(\nabla_{X}^{\prime}(\cdot)\right)=\frac{1}{2} \operatorname{tr}^{\mathbb{R}}\left(\nabla_{X} h\right)-\frac{1}{2} \operatorname{tr}^{\mathbb{R}}\left(J \circ\left(\nabla_{J X} h\right)\right)=\mathcal{L}_{X}\left(\operatorname{tr}^{\mathbb{C}} h\right) .
$$

Moreover, given a local $(J, g)$-unitary frame $\left(e_{i}, J e_{i}\right)$, from (3.1) and (2.11) we get

$$
\begin{aligned}
\operatorname{tr}^{\mathbb{R}}\left(\nabla_{(.)}^{\prime} X\right)= & \frac{1}{2} \sum_{1 \leq i \leq m}\left(g\left(\left(\nabla_{e_{i}} h\right)(X), e_{i}\right)+g\left(\left(\nabla_{J e_{i}} h\right)(X), J e_{i}\right)\right. \\
& \left.-g\left(\left(\nabla_{J e_{i}} h\right)(J X), e_{i}\right)+g\left(\left(\nabla_{e_{i}} h\right)(J X), J e_{i}\right)\right) \\
= & \sum_{1 \leq i \leq m} g\left(\left(\nabla_{e_{i}} h\right)(X), e_{i}\right)+g\left(\left(\nabla_{J e_{i}} h\right)(X), J e_{i}\right) \\
= & \left(\delta^{\nabla} h\right)(X)
\end{aligned}
$$

and so this proves Formula (3.9).

Proposition 3.9 The differential at $g$ in the direction of $h$ of the first Chern-Ricci symmetric endomorphism is given by

$$
g\left(S^{\prime}(X), Y\right)=-g((h \circ S(g))(X), Y)+\frac{1}{2} \operatorname{dd}^{c}\left(\operatorname{tr}^{\mathbb{C}} h\right)(X, J Y) .
$$


Proof Differentiating both sides of $g(S(g)(X), Y)=\tilde{S}(g)(X, J Y)$, we get

$$
g((h \circ S(g))(X), Y)+g\left(S^{\prime}(X), Y\right)=\tilde{S}^{\prime}(X, J Y) .
$$

Therefore, by using (3.5), we get (3.10).

Finally, we are ready to prove the following

Proposition 3.10 The second differential at $g$ in the direction of $\left(h_{1}, h_{2}\right)$ of the Chern-scalar curvature is given by

$$
\begin{gathered}
\left(\operatorname{scal}^{\mathrm{Ch}}\right)^{\prime \prime}=g\left(\operatorname{Hess}_{g}\left(\operatorname{tr}^{\mathbb{C}} h_{1}\right), h_{2}\right)+g\left(\mathrm{~d}\left(\operatorname{tr}^{\mathbb{C}} h_{1}\right), \delta_{g} h_{2}-\delta^{\nabla} h_{2}\right) \\
-g\left(\mathrm{~d}\left(\operatorname{tr}^{\mathbb{C}} h_{1}\right), \vartheta \circ h_{2}\right)+g\left(h_{1}, h_{2} \circ S(g)\right) \\
-\frac{1}{2} g\left(h_{1}, \rho_{g}^{-1}\left(\mathrm{~d} \mathrm{~d}^{c}\left(\operatorname{tr}^{\mathbb{C}} h_{2}\right)\right)\right) .
\end{gathered}
$$

Moreover, if $g$ is Kähler, then

$$
\begin{aligned}
\left(\operatorname{scal}^{\mathrm{Ch}}\right)^{\prime \prime}= & g\left(\operatorname{Hess}_{g}\left(\operatorname{tr}^{\mathbb{C}} h_{1}\right), h_{2}\right)+g\left(h_{1}, \operatorname{Hess}_{g}\left(\operatorname{tr}^{\mathbb{C}} h_{2}\right)\right) \\
& +g\left(h_{1}, h_{2} \circ \operatorname{Ric}(g)\right) .
\end{aligned}
$$

Proof Notice that (3.11) follows directly from (3.6), (3.7), (3.8), (3.9) and (3.10). Assume now that $g$ is Kähler, then

$$
\delta^{\nabla}=\delta_{g}, \quad \vartheta=0, \quad S(g)=\operatorname{Ric}(g) .
$$

Moreover, by (2.10), we get

$$
\begin{aligned}
-\frac{1}{2} g( & \left.h_{1}, \rho_{g}^{-1}\left(\mathrm{dd}^{c}\left(\operatorname{tr}^{\mathbb{C}} h_{2}\right)\right)\right) \\
= & -\frac{1}{2} \sum_{1 \leq \alpha, \beta \leq 2 m} g\left(h_{1}\left(\tilde{e}_{\alpha}\right), \tilde{e}_{\beta}\right) \operatorname{dd}^{c}\left(\operatorname{tr}^{\mathbb{C}} h_{2}\right)\left(\tilde{e}_{\alpha}, J \tilde{e}_{\beta}\right) \\
= & \frac{1}{2} \sum_{1 \leq \alpha, \beta \leq 2 m}\left(g\left(h_{1}\left(\tilde{e}_{\alpha}\right), \tilde{e}_{\beta}\right) g\left(\operatorname{Hess}_{g}\left(\operatorname{tr}^{\mathbb{C}} h_{2}\right)\left(\tilde{e}_{\alpha}\right), \tilde{e}_{\beta}\right)\right. \\
& \left.+g\left(h_{1}\left(\tilde{e}_{\alpha}\right), \tilde{e}_{\beta}\right) g\left(\operatorname{Hess}_{g}\left(\operatorname{tr}^{\mathbb{C}} h_{2}\right)\left(J \tilde{e}_{\alpha}\right), J \tilde{e}_{\beta}\right)\right) \\
= & \sum_{1 \leq \alpha, \beta \leq 2 m} g\left(h_{1}\left(\tilde{e}_{\alpha}\right), \tilde{e}_{\beta}\right) g\left(\operatorname{Hess}_{g}\left(\operatorname{tr}^{\mathbb{C}} h_{2}\right)\left(\tilde{e}_{\alpha}\right), \tilde{e}_{\beta}\right) \\
= & g\left(h_{1}, \operatorname{Hess}_{g}\left(\operatorname{tr}^{\mathbb{C}} h_{2}\right)\right)
\end{aligned}
$$

which concludes the proof of (3.12).

\section{Main results}

Let again $(M, J)$ be a compact, connected, complex manifold of dimension $\operatorname{dim}_{\mathbb{R}} M=2 m$. In this section, we prove our main results, concerning the submersion and local surjectivity properties for the map scal ${ }^{\mathrm{Ch}}: \mathcal{M}_{\mathrm{H}} \rightarrow \mathcal{C}^{\infty}(M, \mathbb{R})$, in view of the relationship between infinitesimal and actual deformations of the Chern-scalar curvature function with respect to a varying metric (see [12] for the Riemannian case). 


\subsection{Submersion points for the Chern-scalar curvature}

We begin this section with the following striaghtforward

Lemma 4.1 For any integer $k \geq m+2$, the map scal ${ }^{\mathrm{Ch}}: \mathcal{M}_{\mathrm{H}}^{k+2} \rightarrow W^{k, 2}(M, \mathbb{R})$ is smooth.

Proof This follows directly by the local formula of the Chern-scalar curvature. More precisely, take a local chart $\left(\mathcal{U}, \xi=\left(z^{1}, \ldots, z^{m}\right)\right)$ of holomorphic coordinates in an open set of $(M, J)$. If we denote by $\Gamma$ the Christoffel symbol of $\nabla$ with respect to $(\mathcal{U}, \xi)$, by using the Koszul Formula for the Chern connection (see e.g. [4, Sect 2.1] for notation), one can directly check that the only non-vanishing components of $\Gamma$ are

$$
\Gamma_{i j}^{k}=g^{k \bar{r}} g_{\bar{r} j, i}, \quad \Gamma_{\bar{i} \bar{j}}^{\bar{k}}=\overline{\Gamma_{i j}^{k}} .
$$

Then, if we denote by $\hat{\Omega}(g) \in \mathcal{C}^{\infty}\left(M, \Lambda^{1,1}\left(T^{*} M\right) \otimes \Lambda^{1,1}\left(T^{*} M\right)\right)$ the totally covariant Chern-curvature, by using (4.1) we can write

$$
\begin{aligned}
\hat{\Omega}(g)= & \Omega_{\bar{j} i \bar{\ell}_{k}} i \mathrm{~d} z^{\bar{j}} \wedge \mathrm{d} z^{i} \otimes i \mathrm{~d} z^{k} \wedge \mathrm{d} z^{\bar{\ell}}, \\
& \Omega_{\bar{j} i \bar{\ell} k}=-\hat{\Omega}\left(\frac{\partial}{\partial z^{i}}, \frac{\partial}{\partial z^{\bar{j}}}, \frac{\partial}{\partial z^{k}}, \frac{\partial}{\partial z^{\bar{\ell}}}\right)=-g_{\bar{\ell} k, \bar{j} i}+g^{s \bar{r}} g_{\bar{r} k, i} g_{\bar{\ell} s, \bar{j}} .
\end{aligned}
$$

Therefore, by the very definition of Chern-scalar curvature, we get

$$
\operatorname{scal}^{\mathrm{Ch}}(g)=2 g^{k \bar{\ell}} g^{i \bar{j}} \Omega_{\bar{j} i \bar{\ell} k}=-2 g^{k \bar{\ell}} g^{i \bar{j}} g_{\bar{\ell} k, \bar{j} i}+2 g^{k \bar{\ell}} g^{i \bar{j}} g^{s \bar{r}} g_{\bar{r} k, i} g_{\bar{\ell} s, \bar{j}} .
$$

Finally, notice that the local formula (4.2) and the multiplicative properties of the Sobolev spaces (see e.g. [1, Thm 4.39]) imply that the map scal ${ }^{\mathrm{Ch}}: \mathcal{M}_{\mathrm{H}}^{k+2} \rightarrow W^{k, 2}(M, \mathbb{R})$ is smooth for any $k \geq m+2$.

For any $g \in \mathcal{M}_{\mathrm{H}}$, we consider the linearized Chern-scalar curvature

$$
\begin{aligned}
& \gamma_{g}: \mathcal{C}^{\infty}\left(M, \operatorname{Sym}^{1,1}(T M)\right) \rightarrow \mathcal{C}^{\infty}(M, \mathbb{R}), \\
& \gamma_{g}(h)=\frac{1}{2}\left(\Delta_{g}\left(\operatorname{tr}^{\mathbb{R}} h\right)+g\left(\mathrm{~d}\left(\operatorname{tr}^{\mathbb{R}} h\right), \vartheta\right)\right)-g(h, S(g))
\end{aligned}
$$

given by (3.6) and its $L^{2}$-adjoint

$$
\begin{aligned}
& \gamma_{g}^{*}: \mathcal{C}^{\infty}(M, \mathbb{R}) \rightarrow \mathcal{C}^{\infty}\left(M, \operatorname{Sym}^{1,1}(T M)\right), \\
& \gamma_{g}^{*}(u)=\frac{1}{2}\left(\Delta_{g} u-g(\mathrm{~d} u, \vartheta)+\left(\mathrm{d}^{*} \vartheta\right) u\right) \operatorname{Id}-u S(g) .
\end{aligned}
$$

For the following result in the Riemannian context, compare [12, Thm 1].

Proposition 4.2 Fix an integer $k \geq m+2$, let $g \in \mathcal{M}_{\mathrm{H}}^{k+2}$ and set $\lambda:=\operatorname{scal}^{\mathrm{Ch}}(g) \in$ $W^{k, 2}(M, \mathbb{R})$. Assume that one of the following is satisfied:

i) $g$ is not first-Chern-Einstein;

ii) $g$ is first-Chern-Einstein and $\frac{\lambda}{m}<\mathrm{d}^{*} \vartheta$.

Then, the map $\mathrm{scal}^{\mathrm{Ch}}: \mathcal{M}_{\mathrm{H}}^{k+2} \rightarrow W^{k, 2}(M, \mathbb{R})$ is a submersion at $g$.

Proof The principal symbol of $\gamma_{g}^{*}$ is clearly injective. Therefore, by means of Remark 2.3, it is sufficient to prove that both (i), (ii) imply that $\gamma_{g}^{*}$ is injective.

Firstly, assume that $g$ is not first-Chern-Einstein. Take $u \in \operatorname{ker}\left(\gamma_{g}^{*}\right)$ and a vector field $X \in \mathcal{C}^{\infty}(M, T M)$ such that $g(X, X)=1$ and set $g(S(g)(X), X)=\frac{\phi}{2 m}$, for some $\phi \in$ $W^{k, 2}(M, \mathbb{R})$. Then, by (4.3) we get

$$
0=2 g\left(\gamma_{g}^{*}(u) X, X\right)=\Delta_{g} u-g(\mathrm{~d} u, \vartheta)+\left(\left(\mathrm{d}^{*} \vartheta\right)-\frac{\phi}{m}\right) u .
$$


Since $k \geq m+2$, by (2.1) the coefficients of (4.4) are of class $\mathcal{C}^{1}$. We then apply [11, Thm 1.17]: it follows that $u \equiv 0$ or there exists an open dense subset $\mathcal{U} \subset M$ such that $u(x) \neq 0$ for any $x \in \mathcal{U}$ and the complement $M \backslash \mathcal{U}$ has zero measure. Assume by contradiction that $u \not \equiv 0$. Then, by (4.3) and (4.4), we obtain

$$
0=\gamma_{g}^{*}(u)=u\left(-S(g)+\frac{\phi}{2 m} \mathrm{Id}\right)
$$

and so

$$
S(g)=\frac{\phi}{2 m} \text { Id } \quad \text { for any } x \in \mathcal{U} .
$$

In particular, this implies that $\phi=\lambda$ and that $g$ is first-Chern-Einstein on the whole manifold $M$, which is not possible by assumption. Then, (i) implies that $\gamma_{g}^{*}$ is injective. to

On the other hand, assume that $S(g)=\frac{\lambda}{2 m} \mathrm{Id}$. Then, the equation $\gamma_{g}^{*}(u)=0$ is equivalent

$$
\Delta_{g} u-g(\mathrm{~d} u, \vartheta)+\left(\left(\mathrm{d}^{*} \vartheta\right)-\frac{\lambda}{m}\right) u=0 .
$$

If (ii) holds true, then the Strong Maximum Principle (see e.g. [18, Thm 4.2]) implies that $u \equiv 0$.

From Proposition 4.2, we get the proofs of Theorem A and Theorem B.

Proof of Theorem $A$ Let $g \in \mathcal{M}_{\mathrm{H}}$, set $\lambda:=\operatorname{scal}^{\mathrm{Ch}}(g) \in \mathcal{C}^{\infty}(M, \mathbb{R})$ and assume that either $g$ is not first-Chern-Einstein, or $g$ is first-Chern-Einstein with $\frac{\lambda}{m}<\mathrm{d}^{*} \vartheta$. Thanks to Proposition 4.2 and the Implicit Function Theorem, we know that, for any integer $k \geq m+2$, the extension of scal ${ }^{\mathrm{Ch}}$ on $\mathcal{M}_{\mathrm{H}}^{k+2}$ is linearization stable at $g$ and maps any neighborhood of $g$ in $\mathcal{M}_{\mathrm{H}}^{k+2}$ onto a neighborhood of $\lambda$ in $W^{k, 2}(M, \mathbb{R})$. Moreover, by means of Theorem 2.2,

$$
W^{k, 2}\left(M, \operatorname{Sym}^{1,1}(T M)\right)=\operatorname{ker}\left(\gamma_{g}\right) \oplus \operatorname{Im}\left(\gamma_{g}^{*}\right) .
$$

It remains to prove the following claim: if $h \in \operatorname{Im}\left(\gamma_{g}^{*}\right) \subset W^{k, 2}\left(M, \operatorname{Sym}^{1,1}(T M)\right)$ and $g_{t}:=g((\operatorname{Id}+t h) \cdot, \cdot \cdot)$, then $\operatorname{scal}^{\mathrm{Ch}}\left(g_{t}\right) \in \mathcal{C}^{\infty}(M, \mathbb{R})$ only if $h \in \mathcal{C}^{\infty}\left(M, \operatorname{Sym}^{1,1}(T M)\right)$.

So, take $h \in \operatorname{Im}\left(\gamma_{g}^{*}\right) \subset W^{k, 2}\left(M, \operatorname{Sym}^{1,1}(T M)\right)$ and assume that scal ${ }^{\mathrm{Ch}}\left(g_{t}\right) \in \mathcal{C}^{\infty}(M, \mathbb{R})$. By differentiating with respect to $t$, it follows that $\gamma_{g}(h) \in \mathcal{C}^{\infty}(M, \mathbb{R})$. By hypothesis, there exists $u \in W^{k+2,2}(M, \mathbb{R})$ such that $h=\gamma_{g}^{*}(u)$. Since $\gamma_{g} \gamma_{g}^{*}$ has injective symbol and $\gamma_{g} \gamma_{g}^{*}(u) \in \mathcal{C}^{\infty}(M, \mathbb{R})$, it follows that $u \in \mathcal{C}^{\infty}(M, \mathbb{R})$. Therefore $h \in \mathcal{C}^{\infty}\left(M, \operatorname{Sym}^{1,1}(T M)\right)$ and the claim follows.

Proof of Theorem B Let us observe that, if one of the conditions stated in Theorem B is satisfied, then $(M, J)$ does not admit any smooth first-Chern-Einstein metric (see [3, Thm 5]). Take $g \in \mathcal{M}_{\mathrm{H}}(\lambda)$. Then, for any $k \geq m+2$, by means of Proposition 4.2 and the Implicit Function Theorem, the preimage $\left(\mathrm{scal}^{\mathrm{Ch}}\right)^{-1}(\lambda)$ inside $\mathcal{M}_{\mathrm{H}}^{k+2}$ is a smooth submanifold in a neighborhood of $g$ with tangent space at $g$ given by $\operatorname{ker}\left(\gamma_{g}\right) \subset W^{k+2,2}\left(M, \operatorname{Sym}^{1,1}(T M)\right)$. This gives rise to a structure of smooth ILH-submanifold on the space $\mathcal{M}_{\mathrm{H}}(\lambda)=\left\{g \in \mathcal{M}_{\mathrm{H}}\right.$ : $\operatorname{scal}^{\mathrm{Ch}}(g)=\lambda$ \} inside $\mathcal{M}_{\mathrm{H}}$.

Remark 4.3 First, we recall that, by [14, Thm 1], in any conformal class of Hermitian metrics, there is a unique Gauduchon metric with volume 1, where being Gauduchon means that the associated $(1,1)$-form $\eta$ satisfies $\mathrm{dd}^{c} \eta^{m-1}=0$. The Gauduchon degree of the conformal class $\{\eta\}$ is then defined as

$$
\Gamma(\{\eta\}):=\int_{M} c_{1}^{\mathrm{BC}}\left(K_{M}^{-1}\right) \wedge \eta^{m-1}=\int_{M} \operatorname{scal}^{\mathrm{Ch}}(\eta) \eta^{m},
$$


and it is equal to the volume of the divisor associated to any meromorphic section of the anticanonical line bundle $K_{M}^{-1}$ by means of the Gauduchon metric, see [15, Sect I.17]. We can now recall the following known facts concerning the condition for $\mathcal{M}_{\mathrm{H}}(\lambda)$ to be non-empty.

- If there exists a conformal class $\{\eta\}$ on $(M, J)$ with $\Gamma(\{\eta\})=0$, then $\mathcal{M}_{\mathrm{H}}(\lambda)$ is non-empty for $\lambda=0$ constant (see [2, Thm 3.1]). Moreover, if $\eta$ is balanced (that is, $\mathrm{d} \eta^{m-1}=0$ ) with $\operatorname{scal}^{\mathrm{Ch}}(\eta)=0$, then $\mathcal{M}_{\mathrm{H}}(\lambda)$ is non-empty if $\lambda \in \mathcal{C}^{\infty}(M, \mathbb{R})$ changes sign and $\int_{M} \lambda \eta^{m}<0$ (see [13, Thm 2.11]).

- If there exists a conformal class $\{\eta\}$ on $(M, J)$ with $\Gamma(\{\eta\})<0$, then $\mathcal{M}_{\mathrm{H}}(\lambda)$ is nonempty for any negative constant $\lambda<0$ (see [2, Thm 4.1]) and for any non-identically zero $\lambda \in \mathcal{C}^{\infty}(M, \mathbb{R})$ such that $\lambda \leq 0$ (see [13, Thm 2.5]). This happens, in particular, when the Kodaira dimension $\operatorname{Kod}(M, J)$ is positive (see [15, Sect I.17]).

- If $M=N \times \Sigma$, where $N$ is a compact complex manifold admitting a conformal class $\{\eta\}$ with $\Gamma(\{\eta\})>0$ and $\Sigma$ is a compact Riemann surface with $\chi(\Sigma)<0$, then $\mathcal{M}_{\mathrm{H}}(\lambda)$ is non-empty for any positive constant $\lambda>0$ (see [2, Prop 5.7]).

- If the Chern-Yamabe conjecture [2, Conj 2.1] has an affirmative answer, then $\mathcal{M}_{\mathrm{H}}(\lambda)$ is non-empty for $\lambda \in I \subset \mathbb{R}$ constant as follows: $I=\mathbb{R}$ when neither $K_{M}$ nor $K_{M}^{-1}$ is pseudo-effective; $I=(0,+\infty)$ when $K_{M}^{-1}$ is pseudo-effective and non-unitary flat; $I=(-\infty, 0)$ when $K_{M}$ is pseudo-effective and non-unitary flat; $I=\{0\}$ when $K_{M}$ is unitary flat (see [30], [32, Thms 1.1, 3.4]).

- Further examples of compact manifolds admitting Hermitian metrics with positive constant Chern-scalar curvature are given by the Hopf surface [17], the homogeneous non-Kähler C-spaces [29], the Hirzebruch surfaces [26], the Bérard-Bergery standard cohomogeneity one complex manifolds [4].

\subsection{Linearization instability and infinitesimal isometries}

We conclude by providing an example of linearization instability. To this aim, we first need the following result due to Fischer-Marsden. For the convenience of the reader, we recall the argument here below.

Lemma 4.4 ([12, Lemma 7.1]) Fix $g_{\mathrm{o}} \in \mathcal{M}_{\mathrm{H}}$. If $\mathrm{scal}^{\mathrm{Ch}}$ is linearization stable at $g_{\mathrm{o}}$, then

$$
\int_{M} u\left(\mathrm{scal}^{\mathrm{Ch}}\right)_{g_{\mathrm{o}}}^{\prime \prime}(h, h) v_{g_{\mathrm{o}}}=0
$$

for any $h \in \operatorname{ker}\left(\gamma_{g_{0}}\right)$, for any $u \in \operatorname{ker}\left(\gamma_{g_{0}}^{*}\right)$.

Proof Fix $h \in \operatorname{ker}\left(\gamma_{g_{0}}\right)$ and $u \in \operatorname{ker}\left(\gamma_{g_{0}}^{*}\right)$. Since scal ${ }^{\text {Ch }}$ is linearization stable at $g_{\mathrm{o}}$, there exists a smooth path $g:(-\epsilon, \epsilon) \rightarrow \mathcal{M}_{\mathrm{H}}$ such that $g(0)=g_{\mathrm{o}}, \dot{g}(0)=h$ and $\mathrm{scal}^{\mathrm{Ch}}(g(t))=$ $\mathrm{scal}^{\mathrm{Ch}}\left(g_{\mathrm{o}}\right)$ for any $-\epsilon<t<\epsilon$. Differentiating this last equation twice, we get

$$
0=\left.\frac{\mathrm{d}^{2}}{\mathrm{~d} t^{2}} \operatorname{scal}^{\mathrm{Ch}}(g(t))\right|_{t=0}=\left(\mathrm{scal}^{\mathrm{Ch}}\right)_{g}^{\prime \prime}(h, h)+\left(\mathrm{scal}^{\mathrm{Ch}}\right)_{g}^{\prime}\left(g^{\prime \prime}(0)\right)
$$


and so, since $\gamma_{g_{0}}^{*}(u)$, we get

$$
\begin{aligned}
0 & =\int_{M} u\left(\mathrm{scal}^{\mathrm{Ch}}\right)_{g}^{\prime \prime}(h, h) v_{g_{0}}+\int_{M} u\left(\mathrm{scal}^{\mathrm{Ch}}\right)_{g}^{\prime}\left(g^{\prime \prime}(0)\right) v_{g_{0}} \\
& =\int_{M} u\left(\mathrm{scal}^{\mathrm{Ch}}\right)_{g}^{\prime \prime}(h, h) v_{g_{0}}+\left\langle u, \gamma_{g_{0}}\left(g^{\prime \prime}(0)\right)\right\rangle_{L^{2}} \\
& =\int_{M} u\left(\mathrm{scal}^{\mathrm{Ch}}\right)_{g}^{\prime \prime}(h, h) v_{g_{0}}
\end{aligned}
$$

which concludes the proof.

In the Theorem C, we consider a Kähler-Einstein metric with positive scalar curvature. Notice that Kähler-Einstein metrics with negative scalar curvature do satisfy the hypothesis of Theorem A, and so they are necessarily linearization stable.

Proof of Theorem C Let $\lambda_{0}:=\operatorname{scal}\left(g_{0}\right)>0$ be the scalar curvature of $g_{0}$. By (3.6), (4.3), (3.12) and by hypothesis, we have

$$
\begin{array}{r}
\gamma_{g_{0}}(h)=\frac{1}{2}\left(\Delta_{g_{0}}\left(\operatorname{tr}^{\mathbb{R}} h\right)-\frac{\lambda_{\mathrm{o}}}{m}\left(\operatorname{tr}^{\mathbb{R}} h\right)\right), \quad \gamma_{g_{\mathrm{o}}}^{*}(u)=\frac{1}{2}\left(\Delta_{g_{0}} u-\frac{\lambda_{\mathrm{o}}}{m} u\right) \mathrm{Id}, \\
\left(\operatorname{scal}^{\mathrm{Ch}}\right)_{g_{\mathrm{o}}}^{\prime \prime}(h, h)=g_{\mathrm{o}}\left(\operatorname{Hess}_{g_{\mathrm{o}}}\left(\operatorname{tr}^{\mathbb{R}} h\right), h\right)+\frac{\lambda_{\mathrm{o}}}{2 m}|h|_{g_{\mathrm{o}}}^{2} .
\end{array}
$$

It is known that the vector space of Killing vector fields is isomorphic to the space of smooth functions $f$ such that $\Delta_{g_{0}} f=\frac{\lambda_{\mathrm{o}}}{m} f$, see e.g. [25, p 96]. Therefore, by hypothesis, $\frac{\lambda_{0}}{m}$ is an eigenvalue of the Laplace-Beltrami operator $\Delta_{g_{0}}$, so that $\operatorname{ker}\left(\gamma_{g_{0}}^{*}\right) \neq\{0\}$. Assume also by contradiction that scal ${ }^{\mathrm{Ch}}$ is linearization stable at $g_{\mathrm{o}}$. Then, by means of (4.5), it follows that

$$
\int_{M} u|h|_{g_{0}}^{2} v_{g_{\mathrm{o}}}=0 \quad \text { for any } u \in \operatorname{ker}\left(\gamma_{g_{\mathrm{o}}}^{*}\right), h \in \mathcal{C}^{\infty}\left(M, \operatorname{Sym}_{0}^{1,1}(T M)\right),
$$

where $\operatorname{Sym}_{0}^{1,1}(T M)$ denotes the subbundle of elements in $\operatorname{Sym}^{1,1}(T M)$ with zero trace. Fix $u \in \mathcal{C}^{\infty}(M, \mathbb{R})$ such that $\Delta_{g_{0}} u=\frac{\lambda_{0}}{m} u$ and $u \not \equiv 0$. By means of the Stokes' Theorem, it follows that

$$
\int_{M} u v_{g_{0}}=0
$$

and so there exists an open ball $B \subset M$ such that $u(x)>0$ for any $x \in B$. Fix $h \in$ $\mathcal{C}^{\infty}\left(M, \operatorname{Sym}_{0}^{1,1}(T M)\right)$ and, up to shrinking $B$, assume that $|h|_{g_{0}}^{2}>0$ on the whole $B$. Pick a smaller ball $B^{\prime} \subset B$ and a function $\psi \in \mathcal{C}^{\infty}(M, \mathbb{R})$ with $\operatorname{supp}(\psi) \subset B$ and such that $\psi(x)=1$ for any $x \in B^{\prime}$. Then, since $\operatorname{tr}^{\mathbb{R}}(\psi h)=0$, by (4.6) we get

$$
0=\int_{M} u|\psi h|_{g_{0}}^{2} v_{g_{0}} \geq \int_{B^{\prime}} u|h|_{g_{\mathrm{o}}}^{2} v_{g_{\mathrm{o}}}>0
$$

which is not possible.

Acknowledgements The authors are warmly grateful to Matteo Focardi and Fabio Podestà for useful discussions and to the anonymous referee for the careful reading of the manuscript.

Open Access This article is licensed under a Creative Commons Attribution 4.0 International License, which permits use, sharing, adaptation, distribution and reproduction in any medium or format, as long as you give appropriate credit to the original author(s) and the source, provide a link to the Creative Commons licence, and indicate if changes were made. The images or other third party material in this article are included in the article's Creative Commons licence, unless indicated otherwise in a credit line to the material. If material is not included in the article's Creative Commons licence and your intended use is not permitted by statutory regulation or exceeds the permitted use, you will need to obtain permission directly from the copyright holder. To view a copy of this licence, visit http://creativecommons.org/licenses/by/4.0/. 


\section{References}

1. Adams, R.A., Fournier, J.J.F.: Sobolev spaces, second edition. Pure and applied mathematics, vol. 140. Elsevier/Academic Press, Amsterdam (2003)

2. Angella, D., Calamai, S., Spotti, C.: On the Chern-Yamabe problem. Math. Res. Lett. 24(3), 645-677 (2017)

3. Angella, D., Calamai, S., Spotti, C.: Remarks on Chern-Einstein Hermitian metrics. Math. Z. 295(3-4), 1707-1722 (2020)

4. Angella, D., Pediconi, F.: On cohomogeneity one Hermitian non-Kähler metrics, preprint version: arXiv:2010.08475

5. Aubin, Th.: Some nonlinear problems in Riemannian geometry, Springer Monographs in mathematics. Springer-Verlag, Berlin (1998)

6. Bérard-Bergery, L.: La courbure scalaire des variétés riemanniennes. Bourbaki seminar, vol. 1979/80, lecture notes in math, vol. 842, pp. 225-245. Springer, Berlin-New York (1981)

7. Berger, M., Ebin, D.: Some decompositions of the space of symmetric tensors on a Riemannian manifold. J. Differ. Geom 3, 379-392 (1969)

8. Berger, M., Gauduchon, P., Mazet, E.: Le spectre d'une variété riemannienne. Lecture notes in mathematics, vol. 194. Springer-Verlag, Berlin-New York (1971)

9. Besse, A.L.: Einstein manifolds, Ergebnisse der Mathematik und ihrer Grenzgebiete, vol. 10. SpringerVerlag, Berlin (1987)

10. Bourguignon, J.-P.: Une stratification de l'espace des structures riemanniennes. Compositio Math. 30, 1-41 (1975)

11. Cheeger, J., Naber, A., Valtorta, D.: Daniele critical sets of elliptic equations. Comm. Pure Appl. Math. 68(2), 173-209 (2015)

12. Fischer, A.E., Marsden, J.E.: Deformations of the scalar curvature. Duke Math. J. 42(3), 519-547 (1975)

13. Fusi, E.: The prescribed Chern scalar curvature problem, arXiv:2105.10220

14. Gauduchon, P.: Le théorème de l'excentricité nulle, C. R. Acad. Sci. Paris Sér. A-B 285(5), A387-A390 (1977)

15. Gauduchon, P.: La 1-forme de torsion d'une variété hermitienne compacte. Math. Ann. 267(4), 495-518 (1984)

16. Gauduchon, P.: Hermitian connections and Dirac operators. Boll. Un. Mat. Ital. B 11(7), 257-288 (1997). (no. 2, suppl)

17. Gauduchon, P., Ivanov, S.: Einstein-Hermitian surfaces and Hermitian Einstein-Weyl structures in dimension 4. Math. Z. 226(2), 317-326 (1997)

18. Goffi, A., Pediconi, F.: A note on the strong maximum principle for fully nonlinear equations on Riemannian manifolds. J. Geom. Anal. 31(8), 8641-8665 (2021)

19. Hebey, E.: Nonlinear analysis on manifolds: sobolev spaces and inequalities. New York University, Courant Institute of Mathematical Sciences, American Mathematical Society, Providence, RI (1999)

20. Ho, P.T.: Results related to the Chern-Yamabe flow. J. Geom. Anal. 31(1), 187-220 (2021)

21. Kazdan, J.L., Warner, F.W.: Curvature functions for compact 2-manifolds. Ann. Math. 99(2), 14-47 (1974)

22. Kazdan, J.L., Warner, F.W.: Scalar curvature and conformal deformation of Riemannian structure. J. Differ. Geom. 10, 113-134 (1975)

23. Kazdan, J.L., Warner, F.W.: Existence and conformal deformation of metrics with prescribed Gaussian and scalar curvatures. Ann. Math. 101(2), 317-331 (1975)

24. Kazdan, J.L., Warner, F.W.: A direct approach to the determination of Gaussian and scalar curvature functions. Invent. Math. 28, 227-230 (1975)

25. Kobayashi, S.: Transformation groups in differential geometry, reprint of the 1972 edition, classics in mathematics. Springer-Verlag, Berlin (1995)

26. Koca, C., Lejmi, M.: Hermitian metrics of constant Chern scalar curvature on ruled surfaces. Kodai Math. J. 3(3), 409-430 (2020)

27. Palais, R: Seminar on the Atiyah-Singer index theorem, with contributions by Atiyah, M.F., Borel, A., Floyd, E.E., Seeley, R.T., Shih, W., Solovay, R., Annals of Mathematics Studies, No. 57 Princeton University Press, Princeton, N.J. (1965)

28. Palais, R.: Seminar on the Atiyah-Singer index theorem. Princeton University Press, Princeton (1965)

29. Podestá, F.: Homogeneous Hermitian manifolds and special metrics. Transform. Groups 23(4), 11291147 (2018)

30. Teleman, A.: The pseudo-effective cone of a non-Kählerian surface and applications. Math. Ann. 335(4), 965-989 (2006) 
31. Tosatti, V.: Non-Kähler Calabi-Yau manifolds, analysis, complex geometry, and mathematical physics: in honor of Duong H. Phong. Contemp. Math. Amer. Math. Soc. Providence, RI 644, 261-277 (2015)

32. Yang, X.-K.: Scalar curvature on compact complex manifolds. Trans. Amer. Math. Soc. 371, 2073-2087 (2019)

Publisher's Note Springer Nature remains neutral with regard to jurisdictional claims in published maps and institutional affiliations. 